\title{
Comparative Analysis of AODV and DSDV Performance in Vanets using NS-2
}

\author{
Anam Mustaqeem \\ Department of Software Engineering University of Engineering and Technology Taxila, Pakistan \\ anam_846@yahoo.com \\ Nadeem Majeed \\ Department of Software Engineering University of Engineering and Technology Taxila, Pakistan \\ Nadeem.majeed@uettaxila.edu.pk \\ Muazzam Maqsood \\ Department of Software Engineering University of Engineering and Technology Taxila, Pakistan \\ muazzammaqsood@yahoo.com
}

\begin{abstract}
Vehicular ad-hoc networks (VANETs) form when vehicles are equipped with devices capable of short-range wireless communication. Vehicular Ad hoc networks involve motion of nodes depending on the mobility model chosen. Three important considerations in simulation of VANETs are mobility models, network simulator and the routing protocols. Selection of appropriate mobility model for evaluating routing protocol leads to efficient simulation results. Performance of routing protocols in VANETs can be measured using four metrics; bandwidth, packet loss, throughput and scalability. This research work is based on the simulation based analysis of Vehicular Ad- hoc networks using NS-2 as the network simulator. Performance evaluation of the protocols is conducted on the basis of four defined metrics and as conclusion is made according to the simulation results.
\end{abstract}

Index Terms - Ad hoc Wireless Networks, MANETs, VANETs, AODV, DSDV

\section{INTRODUCTION}

Wireless technology connects devices without using physical connections. They use radio waves, infrared signals, Bluetooth devices and many more as a medium for communication.

During last few decades, wireless has evolved as an emerging technology in networking field. Continuous progress has opened many new research fields in the context of networking. The aim is at extending the technology to the way where wireless technology may become dominant. Some of the tremendous advantages of wireless networks are high mobility, flexibility and most importantly their low installation cost.

Two main types of wireless networks are

- Infrastructure

- Ad hoc wireless networks
Infrastructure Wireless networks have a set of nodes connected to a central access point. All communication takes place through that central entity. Examples of infrastructure can be mobile communication where connections are oriented towards a central base station, through which nodes broadcast their messages. [1]

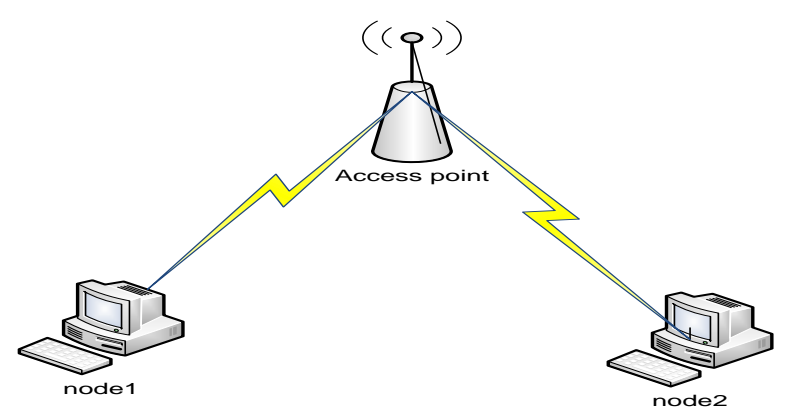

Figure1 Wireless Infrastructure Network

Ad hoc wireless networks support communication of nodes without having any access point or central entity. Nodes are free to communicate within their communication range and their connections can be made on the fly. The forwarding of data packets is based on the routing protocol being used. [2]

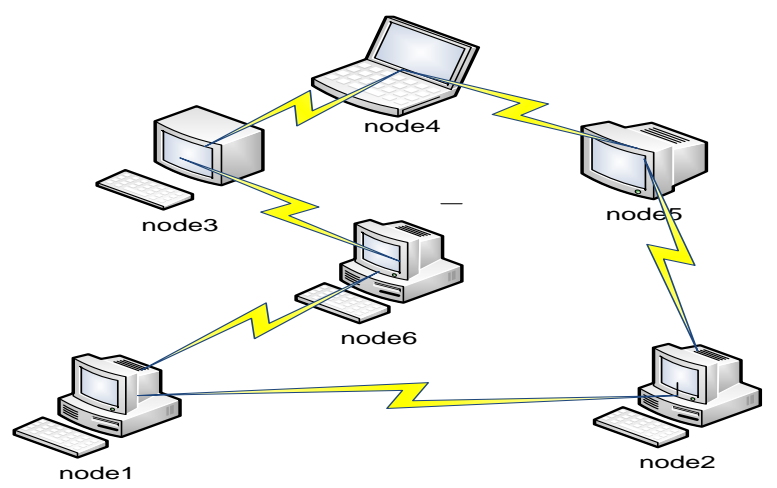

Figure 2 Wireless Ad hoc Network 
Adhoc networks can be further divided into three broader types.

- Mesh Networks

- Sensor Networks

- Mobile Ad hoc Networks

\section{A. Mobile Adhoc Networks(MANETs)}

MANETs are the type of ad hoc networks having random connectivity of nodes in a network. MANETs are considered as self constructing networks as nodes randomly create their own paths while moving in a network topology. [3] Manets have evolved into a new type of network known as VANETs (Vehicular Ad hoc Networks).

\section{B. Vehicular Adhoc Networks(VANETs)}

VANETs are created for the safety of vehicles on roads not using any infrastructure networks. VANETs provide communication between vehicles and vehicle to roadside units. [3] Vehicular Ad hoc Networks (VANETs) are basically characterized by high speed rate of different nodes which ultimately becomes a very important parameter that should be selected very carefully while dealing with different protocols Vehicular Multi-Hop Ad hoc Networks (VANETs) is a vital skill for upcoming expansions of vehicular organizations. Vehicles can communicate with these networks and do not need any infrastructure installation.

This research paper is distributed in four sections; section 1 covers the introduction related to infrastructure, Ad hoc networks, VANETs and MANETs. All the mobility models related to these techniques and their existing algorithms are explained in section 2. There are different types of routing protocols, reactive and proactive. Both these types are explained in chapter 3 along with AODV and DSDV protocols, which are explained along with their diagrams. Different types of simulations scenarios are developed and simulated on ns 2 for AODV and DSDV, their simulation results are explained in section 3 . Analysis and performance evaluation of these two protocols are carried out in section 4 . The paper will be concluded in the last section of conclusion and some suggestion for extension of this research work is proposed in section of future work.

\section{MOBILITY MODELS}

Mobility models describe the pattern of nodes moving in the wireless network scenario. Motion of nodes is very much related to real world scenario; if we replace people with vehicles. These models help in simulation of vehicular ad hoc networks (VANETs). Basically the velocity, geographical position, acceleration and speed of vehicles are well portrayed by the mobility models. [4][5]

Most commonly used mobility models are described below.

\section{A. Random Way Point Model}

Copyright @ 2013 MECS
In Random way point model [4], a node randomly selects a destination point, and moves towards it. Then, after reaching at destination, it stops for a period of time, which is usually known as the "pause time". After pause time, the node again selects another random point to move. This process is continuously repeated until the simulations ends.

node no, $n 1, n 2$

while(simulation)

\{

for destinations $d \&$ nodes $n$;

$n$ takes random $[d]$;

$0<=$ velocity $(n)<=V M A X$;

when $n$ reaches at $d$;

wait for time $t$;

after $t$ ready for next waypoint

\}

simulation ends.

\section{B. City Section Model}

In City section model [4], movement of nodes is limited on a grid road topology, where edges appear to be bi-directional. Each node randomly selects one of the intersections as their destination. Each node moves towards the destination with a constant speed, taking at the most one horizontal and one vertical movement on their way towards destination. Node decides the path which will take shortest travel time towards the destination. Speed of nodes usually depends on the type of road chosen.

Node $n$ \& destination d;

Grid Road topology bidirectional at edges,

Intersections I vertical \$ horizontal;

$N$ chooses random [I] as $d$,

$M A X$ (vertical, horizontal) $=1$,

$N$ calculates shortest time $T$ towards $d$.

$N$ moves towards $d$ with constant speed.

\section{Manhattan Model}

In Manhattan model [5], movement of nodes is very much realistic. Motion of nodes is based on some probability value. Each node moves only one step further at a time. Nodes have many turning points leading from their current location. The probability of choosing path will be equal in all directions i.e. 0.25.

Nodes $n$ \& destination $d$,

Road topology Grid,

Grid contains blocks of equal length $l$,

Node can take MAX[step] $=1$ at any time $T$,

Intersections $I=4$ at each step,

Node can choose any I with probability 0.25 ,

Velocity[n1] is limited by Velocity[nO]

\section{ROUTING PROTOCOLS}

Internet Engineering Task Force (IETF) has proposed several routing protocols for real time implementation of MANETs [6] and VANETs. They can be classified as either reactive, proactive, or hybrid. [7] [8] 


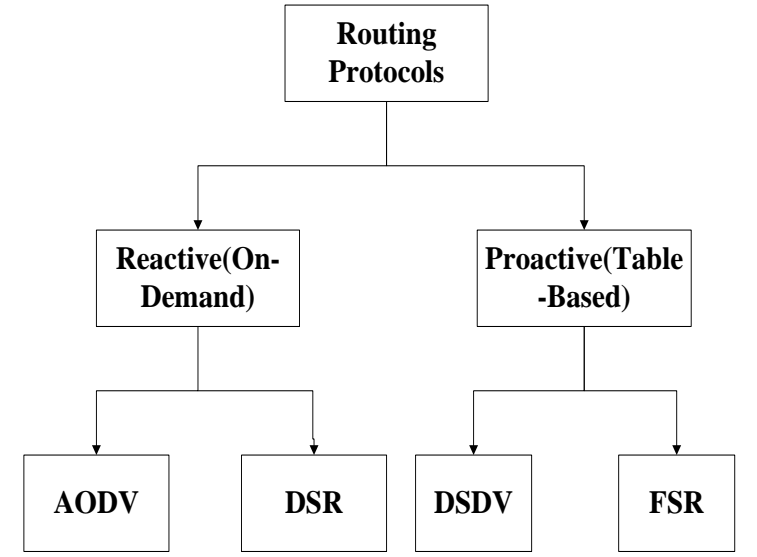

Figure 3 Classification of Routing Protocols for VANETs

\section{A. Reactive Routing Protocol}

In reactive routing protocol [9], connection between the nodes is established only when needed. No pre defined connections exist between the nodes. When a node in a network wants to communicate with any of its surrounding nodes, the sender node sends a Route Request message (RRM) to all the nodes in the network. When the message reaches at the destination node, a Reply message (RM) is sent back to the initiating or the sender node. In this way route is created between the sender and receiving nodes.

There are various protocols that falls under the category of reactive routing protocols. Two of the most widely used protocols that fall under reactive routing in VANETs scenarios are Ad hoc on-Demand Distance Vector Protocol (AODV) and Dynamic Source Routing (DSR).

1) Adhoc on-Demand Distance Vector (AODV)

\section{Protocol:}

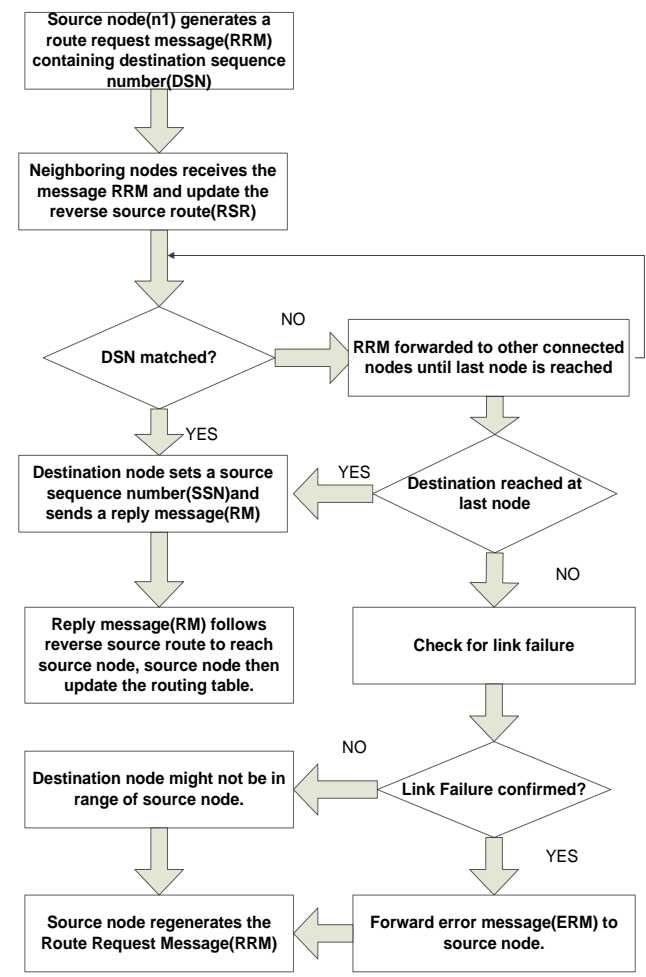

Figure 4 Routing Steps for Message Delivery in AODV
AODV experiences less delay as connection is established only on demand. [10]

2) Simulation Results : Here we present some real time Simulation results for AODV in VANETs. The simulator we have used is NS-2, a discrete network simulator. NS-2 is chosen for simulation because it provides good results for comparison of different routing protocols over both wired and wireless networks.

Ns-2 provides a large amount of libraries for simulation of wireless networks and its routing protocols. The physical layer and the MAC protocol are available for simulation of nodes. [11]

Firstly consider the scenario in which 2 way traffic is maintained using random way point model. Nodes communicate with each other by sending packets. Nodes of one lane can communicate with the other one by maintaining a route to it. All routes in this scenario are generated on demand.

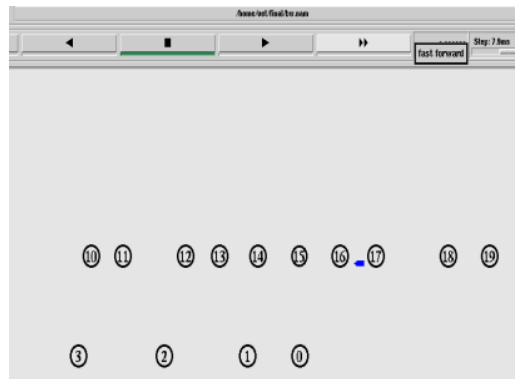

Figure 5 Simulation of AODV using NS-2 Showing Two Way Traffic in VANETs

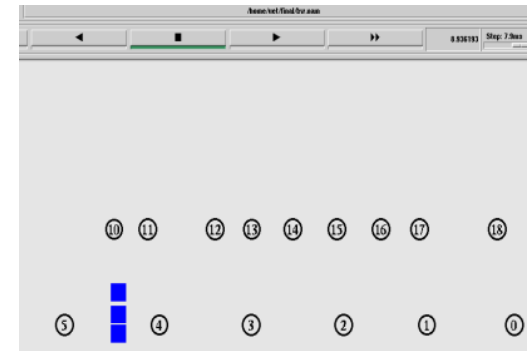

Figure 6 Simulation of AODV using NS-2 Showing Packet Loss in VANETs

As we see there is a packet loss in the above simulation. There can be many reasons for packet loss; link failure, delay, jitter etc. Whenever a packet is lost, source node has to regenerate the message to sort out the route to destination. Sometimes source node will not be informed of packet loss; in such scenario also source node will wait for reply message (RM) for a particular span of time, after which it will regenerate the route request message (RRM). 


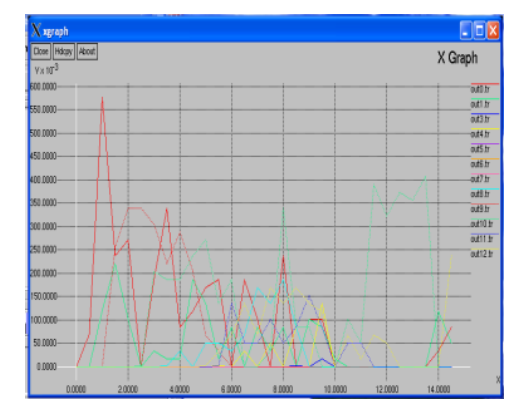

Figure 7 NS-2 Generated Graph for AODV Showing 2 Lane Scenario

The resulted Xgraph represents the bandwidth consumption of nodes at different time intervals. This graph is showing number of bits transmitted per second by each node. $\mathrm{X}$-axis is showing time in Seconds and Y-axis is showing number of bits.

Now consider the case where a four dimensional traffic is maintained in VANETs scenario using Manhattan mobility model. Nodes can change their lanes at intersections. At each intersection, every node has an option of 3 available paths. Node can move to any path with equal probability. Nodes of one lane can communicate with the other one

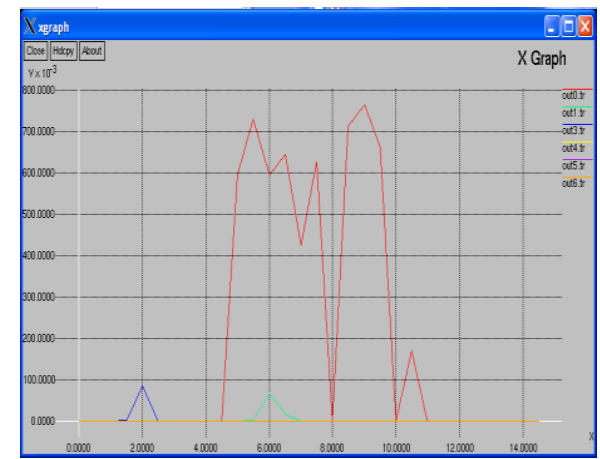

Figure 8 NS-2 Generated Graph for AODV Showing 4 Lane Scenario

\section{B. Proactive Routing Protocols}

These are the routing protocols having routing information available at all times. Proactive means that nodes have predefined routes available to all other nodes in the network. Routing tables are periodically updated with entry of every new node. [12]

As these protocols need to be updated repeatedly, they are not appropriate for larger networks. When there are so many nodes, and each node has to maintain routing entries of all other nodes; this will create a routing overhead which leads to more bandwidth usage. Protocol that fall under reactive routing in VANETs is Dynamic sequence distance vector (DSDV) [13].

1) Dynamic sequence distance vector (DSDV)

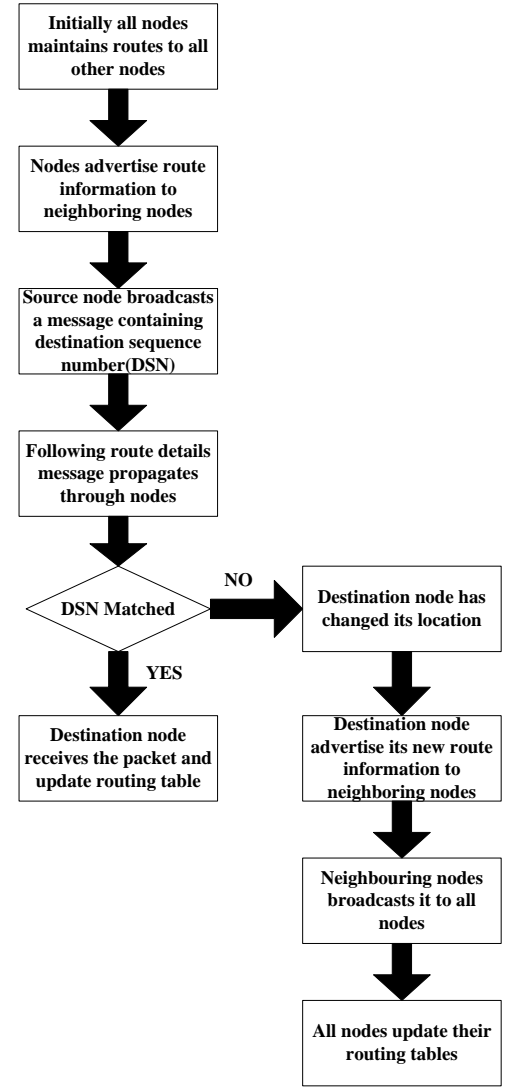

Figure 9 Routing Steps for Message Delivery in DSDV

Simulation Results: Here we present Simulation results for DSDV. Again the network simulator we have used for evaluating the performance of DSDV in VANETs is NS-2.

2 way traffic for DSDV is maintained in VANETs using random way point model. On both lanes a base station is located. Nodes communicate with each other through base station. Nodes of one lane can communicate with the other one through base stations .e.g if a vehicle in lane 1 wants to send a message to vehicle in lane $2 ; \mathrm{V} 1$ will send message signal to BS1 which will forward it to BS2, and then BS2 will forward it to V2.

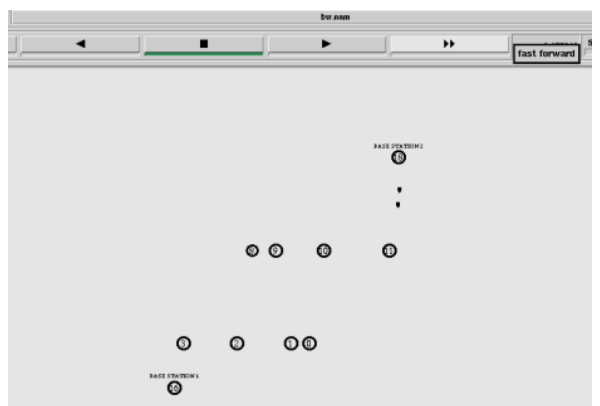

Figure 10 Simulation of DSDV using NS-2 Showing Two Way Traffic in VANETs

The four lane road traffic for DSDV is maintained in VANETs using Manhattan mobility model. On each lane a base station is located. Nodes communicate with each other through base stations. Nodes can change their lanes at intersections. At each intersection, each 
node has an option of 3 available paths. Node can move to any path with equal probability. Nodes of one lane can communicate with the other one through base stations

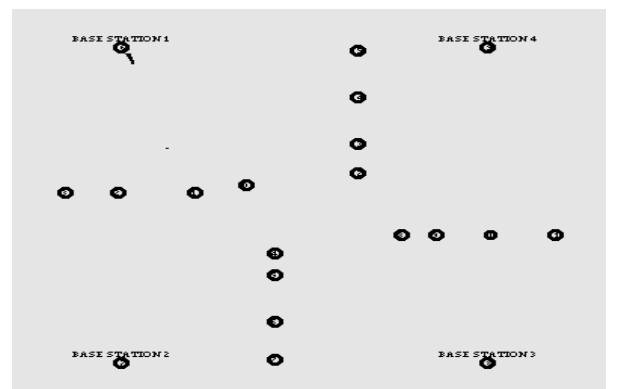

Figure 11 Simulation of DSDV using NS-2 Showing 4 Way Traffic in VANETs

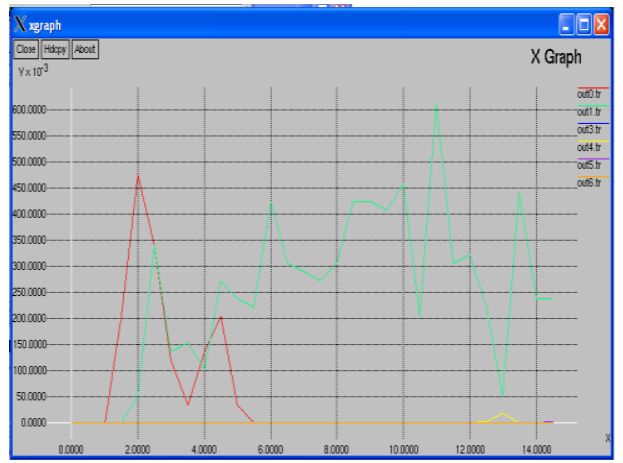

Figure 12 NS-2 Generated Graph for DSDV

\section{ANALYSIS AND FINDINGS}

If we compare the simulation results of AODV and DSDV in VANETs, we can clearly observe their difference. Comparison will be based on following factors.

Bandwidth- In DSDV we have two peaks (red and green) showing more bandwidth consumption. Reason can be routing tables overhead. In DSDV broadcasting concept is used. Base stations continuously broadcast messages to all nodes in the range; this may result in bandwidth wastage especially when nodes are stationary. While in AODV; nodes send messages only to its neighboring nodes which reduce routing overhead as well as bandwidth usage.

Packet Loss-In AODV packet loss occurs frequently due to link failures or unavailability of nodes. Error message informs the source node about packet loss; which after receiving error message regenerates the route request message. This may cause delay. Whereas in DSDV packet loss occurs very often and whenever it does; error message is instantly broadcasted to all nodes in the network.

Throughput-In DSDV the nodes are periodically advertising their routing tables and this will increase in case of higher mobility of nodes. This will eventually decrease the throughput. Whereas in AODV advertisement of routing path is not required, so throughput remains constant.
Scalability- AODV avoids broadcasts while generating route request messages; thereby providing a highly scalable routing protocol. Whereas in DSDV no alternative routes are provided due to which high mobility of nodes may become difficult to handle.

\section{CONCLUSION}

By viewing the above comparison in the context of VANETS we can see that AODV is reactive routing protocol which creates connections only on demand while DSDV provides pre-defined paths for movement of nodes. In DSDV message is broadcasted to all nodes in the networks while in AODV message is forwarded only to the neighboring nodes. These factors make AODV more efficient as compared to DSDV especially for larger VANETs where it becomes difficult to maintain routes to every node. DSDV creates overhead in large VANETs which becomes difficult to manage. So AODV is appropriate for both smaller and larger VANETs as it consumes less bandwidth. But sometimes we do need a base station for broadcasting messages especially when link failure occurs.

\section{FUTURE WORK}

After clearly analyzing the performance metrics of the two routing protocols for VANETs; if we combine the advantages of both AODV and DSDV; we can overcome problem of packet loss occurring in AODV due to link failure or node availability issues. For example if we introduce the concept of broadcasting in AODV; then link failure will not lead to packet loss. Instead whenever link breakage will occur, the message will be broadcasted to all nodes and the alternate path would be considered feasible.

Secondly we can change our network simulator. We can try OMNET as a network simulator for comparative analysis of the two routing protocols in VANETs. And to make it more effective, we can add animations by using some traffic simulators such as SUMO, MOBISIM etc.

\section{ACKNOWLEDGMENT}

Authors are thankful to the department of software engineering from university of engineering and technology Taxila. A deep gratitude for the dean of department for his valuable suggestions and comments on the given research work. A special thanks to Engr. Wajahat Abbas for his guidance.

\section{REFERENCES}

[1] Pentikousis, Qing Zhou, "Design considerations formobility management in future infrastructure networks", Telecom World (ITU WT), 2011 
Technical Symposium at ITU, pp.87 - 92, 24-27 Oct. 2011.

[2] Al Turki, R., Mehmood, R.," Multimedia Ad Hoc Networks: Performance Analysis", Computer Modeling and Simulation, 2008. EMS '08. Second UKSIM European Symposium, pp.561 - 566, 8-10 Sept. 2008

[3] Aamir Hassan "VANET Simulation", Master's Thesis in Electrical Engineering, Technical report, IDE0948, May 2009

[4] Kun-chan Lan , Chien-Ming Chou , "Realistic mobility models for Vehicular Ad hoc Network (VANET) simulations", ITS Telecommunications, 2008, 8th International Conference, pp.362 - 366 , 24-24 Oct. 2008.

[5] Jerome Harri, Fethi Filali and Christian Bonnet," Mobility Models for Vehicular Ad Hoc Networks: A Survey and Taxonomy", Research Report RR06-168, March 5th, 2006, Institute Eurecom Department of Mobile Communications 2229, route des Cretes B.P. 19306904 Sophia-Antipolis FRANCE

[6] Francisco J. Ros ,Pedro M. Ruiz "Implementing a New Manet Unicast RoutingProtocol in NS2" Dept. of Information and Communications Engineering University of Murcia December, 2004

[7] Kwan-Wu Chin, John Judge, Aidan Williams and Roger Kermode, "Implementation Experience with MANET Routing Protocols", Year of Publication: 2002,Sydney Networks and Communications Lab Motorola Australia Research Centre 12 Lord St, Botany, NSW, Australia 2019 fkwchin, johnj, aidan, rkermodeg@arc.corp.mot.com

[8] Krishna Gorantala "Routing Protocols in Mobile Ad-hoc Networks" June 15, 2006, Master's Thesis in Computing Science, $\mathrm{Ume}^{\circ} \mathrm{a}$ University Department of Computing Science SE-901 87 $\mathrm{UME}^{\circ} \mathrm{A}$ SWEDEN

[9] Ing-Chau Chang, Yuan-Fen Wang ; Cheng-Fu Chou, "Efficient VANET Unicast Routing Using Historical and Real-Time Traffic Information", pp.458 - 464, 7-9 Dec. 2011.

[10] C. E. Perkins and E. M. Royer, "Ad-hoc on demand distance vector routing," in Proceedings of the 2nd IEEE Workshop on Mobile Computing Systems and Applications (WMCSA'99), vol. 3, New Orleans, LA, USA, February 1999, pp. 90100.

[11]Gianni A. Di Caro, " Analysis of simulation environments for mobile ad hoc networks" Technical Report No. IDSIA-24-03 December 2003 IDSIA / USI-SUPSI Dalle Molle Institute for Arti_cial Intelligence Galleria 2, 6928 Manno, Switzerland

[12] Zoican, R. , Galatchi, D. ," Analysis and simulation of a Predictable Routing Protocol for VANETs", Electronics and Telecommunications (ISETC), 2010 9th International Symposium, pp.153 - 156, 11-12 Nov. 2010.
[13]Kaur,R., Gaur, M.S. ; Laxmi, V., "A Novel Attack Model Simulation in DSDV Routing", New Technologies, Mobility and Security (NTMS), 2011 4th IFIP International Conference, pp. 1 - 5, 7-10 Feb. 2011.

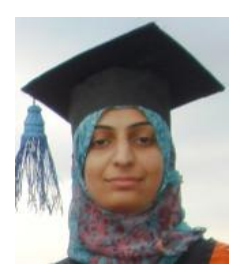

Anam Mustaqeem, is MSc Scholar in Department of Software Engineering at University of Engineering and Technology Taxila. She completed her Bachelor's degree in Software Engineering from University of Engineering and Technology Taxila in 2010. She has done extraordinary work in her four years degree of engineering. She is currently working on multimedia transmission over vehicular Ad-Hoc networks (VANETs). Her areas of interest are digital image processing, Medical Imaging, Software Quality Assurance, Wireless Networks and Ad-Hoc Networks. She is trying hard to bring innovation in above mentioned fields.

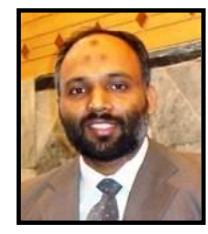

Muhammad Nadeem Majeed is $\mathrm{PhD}$ Scholar in Department of Computer Engineering at University of Engineering \& Technology Taxila. He holds a MS degree in Computer Engineering from Center for Advance Engineering, University of Engineering \& Technology Taxila and has 11 years teaching experience. He is currently serving University of Engineering \& Technology as Assistant Professor and working on Optimized Vertical handoff algorithms in vehicular Ad-hoc network. His research related to Risk management in IT industry of Pakistan which is aimed to aid different managers and team leads to manage the risk in their software development.

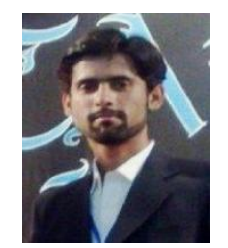

Muazzam Maqsood is an MS Scholar in Department of Software Engineering at University of Engineering \& Technology Taxila. He holds a Bachelor's degree in Software Engineering from University of Engineering \& Technology Taxila and has performed exceptional on his 4 year degree program. His core areas of interest are digital image processing, Software quality assurance, Software Project management and Video Summarization. Muazzam has been striving to bring innovations in the said fields through his research. 